\title{
Output Feedback Controller Design for a Class of MIMO Nonlinear Systems Using High-Order Sliding-Mode Differentiators With Application to a Laboratory 3-D Crane
}

\author{
Weitian Chen, Member, IEEE, and Mehrdad Saif, Senior Member, IEEE
}

\begin{abstract}
This paper addresses the problem of output feedback control design for a class of multi-input-multi-output (MIMO) nonlinear systems where the number of inputs is less than that of outputs. There are two difficulties in this design problem: 1) too few control inputs will not generally allow independent control over all outputs and 2) the state of the system is not available for measurements, and only the outputs are available through measurements. To address the first issue, a practical output feedback control problem is formulated, aiming to regulate only part of the outputs, and a controller structure with two design components in all or some chosen control inputs is proposed. To cope with the second difficulty, the recently developed high-order sliding mode differentiators (HOSMDs) are used to estimate the derivatives of the outputs needed in the controller design. With the derivatives estimated using HOSMDs, an output feedback controller is designed using the backstepping approach. Stability results are established for the designed controller under certain conditions. In order to test the applicability of the proposed output feedback controller in practical industrial problems, experiments are carried out though implementing the controller on a laboratory-scale 3-D crane. The experimental results are presented and reveal the advantage of the proposed controller structure, as well as the effect of controller gain and sampling periods.
\end{abstract}

Index Terms-Backstepping, high-order sliding mode, nonlinear systems, output feedback control.

\section{INTRODUCTION}

B ECAUSE OF ITS robustness to system uncertainties and external disturbances, sliding-mode control (SMC) has received a great deal of attention from the research community [1]-[4] and has found many industrial applications [5]-[8]. The main idea in SMC is to design a proper sliding surface first and then design a switching control law that can force the closed-loop system dynamics to reach and remain on the sliding

Manuscript received January 15, 2008; revised July 31, 2008. Current version published October 31, 2008. This work was supported by the Natural Sciences and Engineering Research Council of Canada through its Discovery Grant Program.

W. Chen was with the School of Engineering Science, Simon Fraser University, Vancouver, BC V5A 1S6, Canada. He is now with the Department of Information Engineering, Research School of Information Sciences and Engineering, The Australian National University, Canberra, ACT 0200, Australia (e-mail: weitian.chen@anu.edu.au).

M. Saif is with the School of Engineering Science, Simon Fraser University, Vancouver, BC V5A 1S6, Canada (e-mail: saif@ensc.sfu.ca).

Color versions of one or more of the figures in this paper are available online at http://ieeexplore.ieee.org.

Digital Object Identifier 10.1109/TIE.2008.2004384 surface that is designed in such a way that control objectives can be met.

SMC enjoys several features such as robustness to disturbances, perfect accuracy after reaching the sliding mode surface, and perhaps finite-time transient period-at least theoretically. However, standard SMC also suffers from certain shortcomings. One of the inherent problems in SMC is the chattering effect caused by high-frequency switching control. Another shortcoming of the traditional SMC methodology is the requirement that the relative degree between the control and the sliding surface needs to be one [9]. This restriction makes it difficult for SMC to be applicable to systems with high relative degrees such as mechanical systems [10] or vehicle control systems [9].

In order to remove some or even all of the restrictions associated with the first-order sliding mode techniques, high-order sling mode design approaches have recently been proposed (see [9]-[11], and the related references therein). According to [9], the idea of high-order SMC (HOSMC) is to first choose a proper $r$ th-order sliding mode determined by $\sigma=\dot{\sigma}=\ddot{\sigma}=$ $\cdots=\sigma^{(r-1)}=0$ and then design a switching control to drive the controlled system to reach and remain on the $r$ th-order sliding mode. According to the definition of $r$ th-order sliding mode, standard SMC aims at achieving a first-order sliding mode. Some examples of second-order sliding mode controllers and observers can be found in [10], [11], and [15], [16], and the related references therein. Examples of HOSMC were proposed in [9] and [17]-[22], to name only a few.

In order to implement HOSMC with the $r$ th-order sliding mode, the derivatives of $\sigma$, i.e., $\dot{\sigma}, \ddot{\sigma}, \ldots, \sigma^{(r-1)}$ have to be used. Because they are not usually measured, they have to be estimated based on the measurement of $\sigma$. One approach for estimating these derivatives is to use high-gain observers [23] [26]. However, high-gain observers only provide asymptotic estimation of the derivatives, and they cannot provide exact reconstruction of the derivatives. Additionally, they do not enjoy finite-time convergence, even for the ideal situation, and in industrial applications, high-gain observers can be quite sensitive to measurement noises [17]. Another approach for estimating the derivatives is to use the recently developed high-order sliding mode differentiator (HOSMD) (see [9], and the related references therein). Ideally, HOSMDs presented in [9] can provide exact reconstruction of the derivatives after a 
finite-time transient period. Moreover, they possess robustness to measurement noises.

The main purpose of this paper is to study the output feedback control design problem for a class of multi-input-multioutput (MIMO) nonlinear systems where the number of inputs is less than that of outputs. Not only is this a challenging problem from a theoretical standpoint, but more importantly, there are many systems in practice that belong to this class of nonlinear systems. Underactuated systems such as overhead cranes [12]-[14] belong to this class of systems. Because of the advantages of the HOSMD over the high-gain observers, in terms of their ability for finite-time exact derivative reconstruction, the output feedback controller uses HOSMDs to estimate the derivatives of the outputs. In the controller design, because there are fewer inputs than outputs, a practical output feedback control problem is formulated, aiming to regulate only part of the outputs. Further, a controller structure with two design components in all or some chosen control inputs is proposed. With the derivatives estimated using HOSMDs, an output feedback controller is designed using the backstepping approach. One difference between the controller in this paper with those highorder sliding mode controllers is that it is designed using the backstepping approach to ensure output tracking and closedloop stability in an asymptotic way rather than to achieve an $r$ th-order sliding mode in finite time. One reason for not working toward to reach an $r$ th-order sliding mode in finite time is that the ideal $r$ th-order sliding mode and finite convergence property will be lost in practical industrial applications due to limitations on sampling rate and the speed of actuation.

Another aim of this paper is to test the practicality of designing an output feedback controller using the estimated output derivatives provided by HOSMDs. This is achieved through experiments on a laboratory-scale 3-D crane system. The experimental results are presented to reveal the advantages of the proposed controller structure, as well as the effect of controller gain and sampling period.

The remainder of this paper is arranged as follows. In Section II, the considered MIMO nonlinear system is introduced, and a realistic output feedback control problem of interest is formulated. In Section III, the sliding mode differentiators given in [9] are presented along with their important properties. In Section IV, a controller structure with two design components in all or some chosen control inputs is proposed. Each design component is designed using the backstepping approach based on the estimation of the derivatives of the outputs provided by HOSMDs. Section V provides experimental results on a laboratory-scale 3-D crane system to illustrate the practicality and performance of the designed output feedback controller using HOSMDs. The experimental results reveal the advantages of the proposed controller structure and the effect of controller gain and sampling period. Finally, concluding remarks are made in the last section.

\section{System of Interest AND PROBlem Formulation}

\section{A. System Description}

Consider a class of MIMO nonlinear systems that is of or can be changed through state transformation into the follow- ing form:

$$
\begin{aligned}
\dot{q}_{i, j} & =q_{i, j+1}, \quad 1 \leq j \leq r_{i}-1 \\
\dot{q}_{i, r_{i}} & =f_{i}(x)+G_{i}(x) u \\
y_{i} & =q_{i, 1}, \quad 1 \leq i \leq p
\end{aligned}
$$

where $u=\left(\begin{array}{lll}u_{1} & \cdots & u_{m}\end{array}\right)^{T} \in R^{m}$ is the input vector, $x=$ $\left(q_{1,1} \cdots q_{1, r_{1}} \cdots q_{p, 1} \cdots q_{p, r_{p}}\right)^{T} \in R^{n}$ is the state vector with $n=r_{1}+\cdots+r_{p}, y \in R^{p}$ is the measured output vector with $y=\left(\begin{array}{llll}y_{1} & y_{2} & \cdots & y_{p}\end{array}\right)^{T}=\left(\begin{array}{llll}q_{1,1} & q_{2,1} & \cdots & q_{p, 1}\end{array}\right)^{T}, f_{i}(x)$ is a scalar function, and $G_{i}(x)=\left(\begin{array}{llll}g_{i, 1} & g_{i, 2} & \cdots & g_{i, m}\end{array}\right), 1 \leq i \leq p$, are row vectors consisting of nonlinear functions of $x$.

It is easy to see that (1) consists of $p$ subsystems of the same form and each subsystem is related to a particular output. For later use, the subsystem related to $y_{i}$ is called the $i$ th subsystem.

In the remaining part of this paper, variables of functions will be dropped for the sake of simplicity wherever appropriate.

Because the controller design for $m \geq p$ is much easier than that for $m<p$, only the case for $m<p$ will be considered in this paper. Let $G(x)=\left(\begin{array}{lll}G_{1}^{T} & \cdots & G_{p}^{T}\end{array}\right)^{T}=\left(g_{i, j}\right)_{p \times m}$ and $F(x)=\left(f_{1} \cdots f_{p}\right)^{T}$, and the following assumption is made.

[A1] All the elements in $F(x)$ and $G(x)$ are bounded for bounded $x$, and $\operatorname{rank}(G)=m$ for all $x$ in the region of interest.

\section{B. Problem Formulation}

When $m<p$, it is in general very difficult, if not impossible, to design an output feedback controller to regulate all the outputs (i.e., to make the outputs independently track desired independent reference signals). Actually, in this case, even the stabilization problem may become very hard to solve. Because of this restriction, a realistic output feedback control problem is formulated as follows.

Output Feedback Control Problem: Under Assumption A1 and the assumption that $m<p$, the objective is to design, if possible, an output feedback controller such that it can regulate a chosen group of outputs (e.g., $m_{\mu}$ outputs, where $1 \leq m_{\mu} \leq$ $m$ ) and ensure that all the signals in the closed-loop system are bounded at the same time.

Without loss of generality, assume that the chosen group of $m_{\mu}$ outputs are the first $m_{\mu}$ outputs, i.e., $y_{1}, y_{2}, \ldots, y_{m_{\mu}}$. The problem becomes how an output feedback controller can be designed such that it can make $y_{1}, y_{2}, \ldots, y_{m_{\mu}}$ track desired reference signals and ensure that all the signals in the closedloop system are bounded at the same time.

Denote the desired reference signals as $y_{1, r}(t), y_{2, r}(t), \ldots$, $y_{m_{\mu}, r}(t)$, and the following assumption is made on them.

[A2] For each $1 \leq i \leq m_{\mu}, y_{i, r}$ is $r_{i}$ times differentiable, and $y_{i, r}^{\left(r_{i}\right)}(t)$ is bounded.

\section{HOSMD AND ITS PROPERTIES}

Since HOSMDs will be used to provide the estimates of the derivatives of the outputs for the purpose of controller design, an HOSMD presented in [9] will be introduced along with its properties in this section before we move on to controller design. 


\section{A. $H O S M D$}

Let $s(t)=s_{0}(t)+n(t)$ be a function on $[0, \infty)$, where $s_{0}(t)$ is an unknown base function with the $n$th derivatives having a Lipschitz constant $L$, and $n(t)$ is a bounded Lebesguemeasurable noise with unknown features. The problem of high-order sliding-mode robust differentiator design is to find real-time robust estimations of $\dot{s}_{0}(t), \ddot{s}_{0}(t), \ldots, s_{0}^{(n)}(t)$, being exact when $n(t)=0$. An HOSMD proposed in [9] takes on the following form:

$$
\begin{aligned}
\dot{z}_{0} & =v_{0} \\
v_{0} & =-\lambda_{0}\left|z_{0}-s(t)\right|^{n /(n+1)} \operatorname{sign}\left(z_{0}-s(t)\right)+z_{1} \\
\dot{z}_{1} & =v_{1} \\
v_{1} & =-\lambda_{1}\left|z_{1}-v_{0}\right|^{(n-1) / n} \operatorname{sign}\left(z_{1}-v_{0}\right)+z_{2} \\
& \vdots \\
\dot{z}_{n-1} & =v_{n-1} \\
v_{n-1} & =-\lambda_{n-1}\left|z_{n-1}-v_{n-2}\right|^{1 / 2} \operatorname{sign}\left(z_{n-1}-v_{n-2}\right)+z_{n} \\
\dot{z}_{n} & =-\lambda_{n} \operatorname{sign}\left(z_{n}-v_{n-1}\right)
\end{aligned}
$$

where $\lambda_{0}, \lambda_{1}, \ldots, \lambda_{n}$ are positive design parameters.

\section{B. Properties of the HOSMD}

Regarding the HOSMD given in (2), the following three results have been proved [9].

Theorem 1: If $n(t)=0$ and all the parameters are chosen properly, then after a finite transient, the following equalities are true:

$$
z_{0}=s_{0}(t) ; \quad z_{i}=v_{i-1}=s_{0}^{(i)}(t), \quad i=1,2, \ldots, n .
$$

Theorem 2: If $|n(t)|=\left|s(t)-s_{0}(t)\right| \leq \epsilon$ and all the parameters are chosen properly, then after a finite transient, the following inequalities are obtained:

$$
\begin{aligned}
\left|z_{i}-s_{0}^{(i)}(t)\right| \leq \mu_{i} \epsilon^{(n-i+1) /(n+1)}, & i=0,1, \ldots, n \\
\left|v_{i}-s_{0}^{(i+1)}(t)\right| & \leq \nu_{i} \epsilon^{(n-i) /(n+1)}, \quad i=0,1, \ldots, n-1
\end{aligned}
$$

where $\mu_{i}, i=0,1, \ldots, n$, and $\nu_{i}, i=0,1, \ldots, n-1$, are some positive constants that depend only on the parameters of the differentiator.

Consider the discrete-sampling case when $z_{0}(t)-s(t)$ is replaced by $z_{0}\left(t_{j}\right)-s\left(t_{j}\right)$ on $\left[t_{j}, t_{j+1}\right)$ with $\tau=t_{j+1}-t_{j}$.

Theorem 3: Let $\tau$ be the constant sampling time. If $n(t)=0$ and all the parameters are chosen properly, then after a finite transient, the following inequalities are obtained:

$$
\begin{gathered}
\left|z_{i}-s_{0}^{(i)}(t)\right| \leq \mu_{i} \tau^{n-i+1}, \quad i=0,1, \ldots, n \\
\left|v_{i}-s_{0}^{(i+1)}(t)\right| \leq \nu_{i} \tau^{n-i}, \quad i=0,1, \ldots, n-1 .
\end{gathered}
$$

Remark 1: Theorem 1 shows that finite-time exact reconstruction of the derivatives of $s(t)$ can be achieved-at least ideally. Theorem 2 states that the HOSMD can provide good estimates for the derivatives only if the measurement noises are small enough. Theorem 3 reveals that the estimation accuracy of the derivatives depends also heavily on the sampling period, even for the noise-free case, and the faster the sampling is, the better the estimation accuracy of the derivatives becomes.

\section{Output Feedback Controller Design}

In this section, first, a controller structure with two design components in all or some chosen control inputs will be proposed. Then, each component will be designed using the backstepping approach, assuming all states are available, and the stability of the designed state feedback controller is analyzed. Finally, by the use of the estimated derivatives of the outputs offered by HOSMDs, an output feedback controller is provided, and under certain conditions, stability results will be given.

Define $G_{\mathrm{tr}}(x)=\left(\begin{array}{lllll}G_{1}^{T} & \cdots & G_{m_{\mu}}^{T} & \cdots & G_{m}^{T}\end{array}\right)^{T}, \quad F_{\mathrm{tr}}(x)=$ $\left(\begin{array}{lllll}f_{1} & \cdots & f_{m_{\mu}} & \cdots & f_{m}\end{array}\right)^{T}, \bar{G}_{\mathrm{tr}}(x)=\left(\begin{array}{lll}G_{m+1}^{T} & \cdots & G_{p}^{T}\end{array}\right)^{T}$, and $\bar{F}_{\mathrm{tr}}(x)=\left(f_{m+1} \cdots f_{p}\right)^{T}$. The following assumption is needed for the controller design.

[A3] For all $x$ in the region of interest, $G_{\mathrm{tr}}$ is bounded and invertible, and $\operatorname{rank}\left(\bar{G}_{\text {tr }}\right)=p-m$.

\section{A. Controller Structure}

In order to solve the formulated output feedback control problem, the following controller structure is proposed:

$$
u=u_{\mathrm{tr}}+u_{\mathrm{sta}}
$$

where $u_{\mathrm{tr}}=\left(\begin{array}{lll}u_{\mathrm{tr}, 1} & \cdots & u_{\mathrm{tr}, m}\end{array}\right)^{T}$ is a design component that is introduced to make the outputs $y_{1}, y_{2}, \ldots, y_{m_{\mu}}$ track the desired reference signals $y_{1, r}(t), y_{2, r}(t), \ldots, y_{m_{\mu}, r}(t)$ asymptotically when $u_{\mathrm{sta}}=0$, and $u_{\mathrm{sta}}=\left(\begin{array}{llll}u_{\mathrm{sta}, 1} & \cdots & u_{\mathrm{sta}, m}\end{array}\right)^{T}$ is another design component that is introduced to ensure that all the closed-loop signals are bounded.

The detailed design of $u_{\mathrm{tr}}$ and $u_{\mathrm{sta}}$ is given in Section IV-B.

\section{B. State Feedback Controller}

In this subsection, the design of $u_{\mathrm{tr}}$ and $u_{\text {sta }}$ using state feedback will be proposed, and the stability of the designed controller is analyzed under certain conditions.

Define $U(x)=\left(U_{1}(x) U_{2}(x) \cdots U_{p}(x)\right)^{T}=F+G u$, then (1) can be rewritten as

$$
\begin{aligned}
\dot{q}_{i, j} & =q_{i, j+1}, \quad 1 \leq j \leq r_{i}-1 \\
\dot{q}_{i, r_{i}} & =U_{i} \\
y_{i} & =q_{i, 1}, \quad 1 \leq i \leq p .
\end{aligned}
$$

For the design purpose, let us introduce a group of reference signals for $y_{m_{\mu}+1}, \ldots, y_{p}$ as $y_{m_{\mu}+1, r}, \ldots, y_{p, r}$. Unlike $y_{1, r}(t), y_{2, r}(t), \ldots, y_{m_{\mu}, r}(t)$, which are predetermined by control objectives, $y_{m_{\mu}+1, r}, \ldots, y_{p, r}$ are left to be chosen freely in order to provide the designers with more freedom. The designers might want to choose some particular groups of $y_{m_{\mu}+1, r}, \ldots, y_{p, r}$ that will make the controller design easier to ensure that all the signals in the closed-loop system are 
bounded. In order to illustrate this point clearly, consider a linear system described as

$$
\begin{aligned}
& \dot{x}_{1}=u_{1} \quad \dot{x}_{2}=u_{2} \quad \dot{x}_{3}=x_{4} \quad \dot{x}_{4}=u_{2} \\
& y_{1}=x_{1} \quad y_{2}=x_{2} \quad y_{3}=x_{3} \text {. }
\end{aligned}
$$

For this system, choose $m_{\mu}=1$, which means that one would like only to make $y_{1}$ to track any reference signal $y_{1, r}$. If $y_{2, r}=1$ is also fixed and $u_{2}=-\left(x_{2}-1\right)$ is chosen in order to track $y_{2, r}$, it is easy to check that $x_{3}$ will go unbounded for any $x_{4}(0)-x_{2}(0)+1 \neq 0$ and for any $u_{1}$. However, if $y_{2, r}$ and $y_{3, r}$ are allowed to be chosen freely and $u_{2}=-2 x_{3}-3 x_{4}$ is chosen, it is easy to show that $x_{3}$ and $x_{4}$ both tend to zero exponentially with $x_{2}$ being bounded. All signals in the closed loop can be made bounded if $u_{1}$ is designed properly to make $y_{1}$ track $y_{1, r}$.

Remark 2: The above example showed that for some systems, the proper choice of $y_{m_{\mu}+1, r}, \ldots, y_{p, r}$, together with proper controller design will help stabilize the whole system. In order to do this, it is generally required that $m_{\mu}<m$. If $m_{\mu}=m$, all the controls are fixed by the proposed controller design. The stability of the overall closed-loop system would be system dependent, and there is generally no guarantee that the system is not unstable. That is the reason that some control design freedom is required to be left in this paper. However, a systematic design approach for those free chosen reference signals is unable to be provided at the present time, which is quite interesting and requires further research.

Remark 3: It might be possible to define a new output as a proper combination of the actual outputs to make the guaranteed stabilization for some systems. However, whether this can be done for all systems of the form (1) and how such a new output can be defined are not trivial and require further investigations.

For the time being, let us assume that $x=\left(q_{1,1} \quad \cdots\right.$ $\left.q_{1, r_{1}} \cdots q_{p, 1} \cdots q_{p, r_{p}}\right)^{T}$ is available, and that $U_{i}$ is free to design for all $i$. Then, for each $i, U_{i}$ can be designed as follows using the backstepping approach (since the backstepping approach is now quite standard, for the detailed design procedure, the interested readers are referred to [27]):

$$
\begin{aligned}
U_{i}=-\left(c_{i, r_{i}}+1\right) \xi_{i, r_{i}}-\xi_{i, r_{i}-1} \\
+\sum_{j=1}^{r_{i}-1} \frac{\partial \alpha_{i, r_{i}-1}}{\partial q_{i, j}} q_{i, j+1}+\sum_{j=0}^{r_{i}-1} \frac{\partial \alpha_{i, r_{i}-1}}{\partial y_{i, r}^{(j)}} y_{i, r}^{(j+1)}
\end{aligned}
$$

where $\xi_{i, k}, 1 \leq k \leq r_{i}$, and $\alpha_{i, k}, 1 \leq k \leq r_{i}-1$, are defined as

$$
\begin{aligned}
\xi_{i, 1}= & q_{i, 1}-y_{i, r} \\
\alpha_{i, 1}= & -c_{i, 1} \xi_{i, 1}+\dot{y}_{i, r} \\
\xi_{i, k}= & q_{i, k}-\alpha_{i, k-1}, \quad 2 \leq k \leq r_{i} \\
\alpha_{i, k}= & -c_{i, k} \xi_{i, k}-\xi_{i, k-1}+\sum_{j=1}^{k-1} \frac{\partial \alpha_{i, k-1}}{\partial q_{i, j}} q_{i, j+1} \\
& +\sum_{j=0}^{k-1} \frac{\partial \alpha_{i, k-1}}{\partial y_{i, r}^{(j)}} y_{i, r}^{(j+1)}, \quad 2 \leq k \leq r_{i}-1
\end{aligned}
$$

and $y_{i, r}^{(j)}$ is the $j$ th-order derivative of $y_{i, r}$, with $y_{i, r}^{(0)}=y_{i, r}$, and $c_{i, j}, 1 \leq i \leq p, 1 \leq j \leq r_{i}$, are positive constants that can be chosen freely.

For each $i$, if $U_{i}$ was to be applied to the $i$ th subsystem, according to the backstepping approach, one would be able to prove that $\lim _{t \rightarrow \infty} \xi_{i, 1}=0$ and establish the boundness of $\xi_{i, k}$, $1 \leq k \leq r_{i}$, or equivalently, $q_{i, k}, 1 \leq k \leq r_{i}$. The formulated output feedback control problem would thus be solved.

However, because $m<p$, it is generally not possible to achieve all $U_{i}, 1 \leq i \leq p$, through the design of $m$ controls, i.e., $u_{i}, 1 \leq i \leq m$. This makes the controller design much more complicated.

In order to solve the formulated output feedback control problem, one needs to design $u_{\mathrm{tr}}$ to achieve $U_{\mathrm{tr}}$, where $U_{\mathrm{tr}}=$ $\left(U_{1} \cdots U_{\mu} \cdots U_{m}\right)^{T}$.

To achieve $U_{\mathrm{tr}}, u_{\mathrm{tr}}$ is designed as

$$
u_{\mathrm{tr}}=G_{\mathrm{tr}}^{-1}\left(U_{\mathrm{tr}}-F_{\mathrm{tr}}\right)
$$

where $G_{\mathrm{tr}}^{-1}$ is the inverse of $G_{\mathrm{tr}}$.

Now, all that remains in the controller design is to design $u_{\text {sta }}$.

Since $\operatorname{rank}\left(\bar{G}_{\mathrm{tr}}\right)=p-m$, it can be assumed without loss of generality that the first $p-m$ columns of $\bar{G}_{\mathrm{tr}}$ are independent. Denote $\bar{G}_{\mathrm{tr}}=\left(\bar{G}_{\mathrm{tr}, 1} \bar{G}_{\mathrm{tr}, 2}\right)$, where $\bar{G}_{\mathrm{tr}, 1}$ consists of the first $p-m$ columns of $\bar{G}_{\mathrm{tr}}$, and $\bar{G}_{\mathrm{tr}, 2}$ consists of the remaining columns.

Denote $\bar{U}_{\mathrm{tr}}=\left(\begin{array}{lll}U_{m+1} & \cdots & U_{p}\end{array}\right)^{T}$, and define $\bar{u}_{p-m}=$ $\bar{G}_{\mathrm{tr}, 1}^{-1} \bar{U}_{\mathrm{tr}}=\left(\bar{u}_{m+1} \cdots \bar{u}_{p}\right)^{T}$. Then, because the primary purpose of designing $u_{\text {sta }}$ is to ensure that the signals in the closed loop are bounded, the principle of the design of $u_{\text {sta }}$ is to make the linearized parts of the last $p-m$ subsystems stable. To this end, $u_{\text {sta }}$ is designed as

$$
u_{\text {sta }}=\left(u_{p-m}^{T} 0\right)^{T}
$$

where 0 is zero vector with a compatible dimension, and $u_{p-m}=\left(\operatorname{sat}_{M}\left(\bar{u}_{m+1}\right) \quad \cdots \quad \operatorname{sat}_{M}\left(\bar{u}_{p}\right)\right)^{T}$ with function $\operatorname{sat}_{M}\left(\bar{u}_{j}\right)$ being defined as

$$
\begin{aligned}
\operatorname{sat}_{M}\left(\bar{u}_{j}\right) & =\bar{u}_{j}, & & \text { if }\left|\bar{u}_{j}\right| \leq M \\
& =M, & & \text { if } \bar{u}_{j}>M \\
& =-M, & & \text { if } \bar{u}_{j}<-M
\end{aligned}
$$

where $M$ is a positive design constant. The saturation functions are introduced to prove the stability of the closed-loop system, and $M$ can be chosen to be as large as possible.

Define $\bar{x}^{1}=\left(q_{1,1} \cdots q_{1, r_{1}} \cdots q_{m, 1} \cdots q_{m, r_{m}}\right)^{T}$ and $\bar{x}^{2}=$

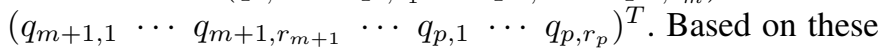
definitions, one more assumption is made as follows.

[A4] When the state feedback controller given in (6) and (11)-(13) is applied to (1), $\bar{x}^{2}=\left(q_{m+1,1} \quad \cdots\right.$

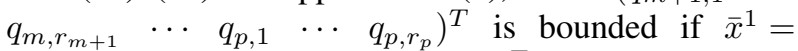
$\left(q_{1,1} \cdots q_{1, r_{1}} \cdots q_{m, 1} \cdots q_{m, r_{m}}\right)^{T}$ is bounded.

At this juncture, we are ready to state stability results related to the state feedback controller.

Theorem 4: Given A1-A4, and assuming that the state vector $x$ is available, if the state feedback controller given in 
(6) and (11)-(13) is applied to (1), then the following statements hold.

1) The output tracking error, i.e., $\left|y_{i}-y_{i, r}\right|$, will enter a small neighborhood of zero for any $1 \leq i \leq m$, and moreover, $\lim _{t \rightarrow \infty}\left|y_{i}-y_{i, r}\right|=0$ for any $1 \leq i \leq m$ if $\lim _{t \rightarrow \infty} u_{\text {sta }}=0$.

2) All closed-loop signals are bounded.

Proof: Consider only the subsystem related to $\bar{x}^{1}=$ $\left(q_{1,1} \cdots q_{1, r_{1}} \cdots q_{m, 1} \cdots q_{m, r_{m}}\right)^{T}$.

With the definitions of $F(x), F_{\mathrm{tr}}(x), G(x), G_{\mathrm{tr}}(x), u_{\mathrm{tr}}$, and $U_{\mathrm{tr}}(x)$, and using (6), one gets

$$
F_{\mathrm{tr}}(x)+G_{\mathrm{tr}}(x) u=U_{\mathrm{tr}}(x)+G_{\mathrm{tr}}(x) u_{\mathrm{sta}} .
$$

For the sake of simplicity, denote $N=\left(N_{1} \cdots N_{m}\right)^{T}=$ $G_{\mathrm{tr}} u_{\mathrm{sta}}$. Because $G_{\mathrm{tr}}(x)$ is bounded for all $x$ by assumption, and $u_{\text {sta }}$ is designed to be bounded, $N_{i}, 1 \leq i \leq m$, are bounded.

By the definitions of $u_{\mathrm{tr}}$ and $U_{\mathrm{tr}}(x)$, and after applying (6) and (11)-(13) to (1), the subsystem related to $\bar{x}^{1}$ becomes

$$
\begin{aligned}
\dot{q}_{i, j} & =q_{i, j+1}, & & 1 \leq j \leq r_{i}-1 \\
\dot{q}_{i, r_{i}} & =U_{i}+N_{i}, & & 1 \leq i \leq m .
\end{aligned}
$$

For any $1 \leq i \leq m$, by substituting $U_{i}$ defined in (9) and (10) into (15), the following equations can be derived:

$$
\begin{aligned}
\dot{\xi}_{i, 1} & =-c_{i, 1} \xi_{i, 1}+\xi_{i, 2} \\
\dot{\xi}_{i, j} & =-c_{i, j} \xi_{i, j}+\xi_{i, j+1}-\xi_{i, j-1}, \quad 2 \leq j \leq r_{i}-1 \\
\dot{\xi}_{i, r_{i}} & =-\left(c_{i, r_{i}}+1\right) \xi_{i, r_{i}}-\xi_{i, r_{i}-1}+N_{i} .
\end{aligned}
$$

Choosing a Lyapunov function as $V_{i}=1 / 2 \sum_{j=1}^{r_{i}} \xi_{i, j}^{2}$, and differentiating it along (16), one obtains

$$
\dot{V}_{i}=-\sum_{j=1}^{r_{i}} c_{i, j} \xi_{i, j}^{2}-\xi_{i, r_{i}}^{2}+N_{i} \xi_{i, r_{i}}
$$

Denote $\quad c_{i, \min }=\min \left\{c_{i, j} \mid 1 \leq j \leq r_{i}\right\} \quad$ and $\quad \bar{N}_{i}=N_{i}^{2} / 4$. Then, it follows from (17) that

$$
\dot{V}_{i} \leq-2 c_{i, \min } V_{i}+\bar{N}_{i} .
$$

Since $N_{i}$ is bounded, $c_{i, j}, 1 \leq j \leq r_{i}$, can be chosen such that

$$
\frac{\left|\bar{N}_{i}\right|}{c_{i, \min }} \leq \epsilon_{i}
$$

where $\epsilon_{i}$ is a small constant.

Then, the following can be derived:

$$
\begin{aligned}
V_{i}(t) & \leq e^{-2 c_{i, \min } t}\left(V_{i}(0)+\int_{0}^{t} \bar{N}_{i} e^{2 c_{i, \min } \tau} d \tau\right) \\
& \leq e^{-2 c_{i, \min } t}\left(V_{i}(0)+\epsilon_{i} c_{i, \min } \int_{0}^{t} e^{2 c_{i, \min } \tau} d \tau\right) \\
& =e^{-2 c_{i, \min } t}\left(V_{i}(0)-\frac{\epsilon_{i}}{2}\right)+\frac{\epsilon_{i}}{2} .
\end{aligned}
$$

This implies that

$$
\left|\xi_{i, 1}\right| \leq \sqrt{2\left(V_{i}(0)-\frac{\epsilon_{i}}{2}\right) e^{-2 c_{i, \min } t}+\epsilon_{i}} .
$$

Equation (21) means that the tracking error $\left|y_{i}-y_{i, r}\right|=\left|\xi_{i, 1}\right|$ will eventually enter the neighborhood of zero, namely, $\mid y_{i}-$ $y_{i, r} \mid \leq \sqrt{\epsilon_{i}}$.

Moreover, if $\lim _{t \rightarrow \infty} u_{\text {sta }}=0$, it follows from Assumptions A1-A3 that $\lim _{t \rightarrow \infty} N_{i}=0$, and thus, $\lim _{t \rightarrow \infty} \epsilon_{i}=0$. This, together with (21), implies that $\lim _{t \rightarrow \infty}\left|y_{i}-y_{i, r}\right|=0$.

Based on (20), it can also be concluded that $\xi_{i, j}, 1 \leq j \leq r_{i}$, and, thus, $q_{i, j}, 1 \leq j \leq r_{i}$, are bounded. Since this conclusion is true for any $1 \leq i \leq m$, it is proved that $\bar{x}^{1}$ is bounded. This fact, together with Assumption A4, implies that $\bar{x}^{2}$ is also bounded. Therefore, the state vector $x$ of the closedloop system is bounded. The boundness of $x$, together with Assumptions A1-A3, implies that $u_{\mathrm{tr}}$ is bounded, which proves that the control $u$ is also bounded. This completes the proof.

Based on the results in Theorem 4, it is easy to prove the following.

Theorem 5: Assume that the second design component is chosen to be zero, i.e., $u_{\text {sta }}=0$, and that the assumptions of Theorem 4 are all satisfied. If the state feedback controller given in (6) and (11)-(13) is applied to (1), then the following statements hold.

1) $\lim _{t \rightarrow \infty}\left|y_{i}-y_{i, r}\right|=0$ for any $1 \leq i \leq m$.

2) All closed-loop signals are bounded.

Proof: Because $u_{\text {sta }}=0$, following the arguments in the proof of Theorem 4 , for any $1 \leq i \leq m$, one can reach

$$
\dot{V}_{i} \leq-\sum_{j=1}^{r_{i}} c_{i, j} \xi_{i, j}^{2} .
$$

This implies that $\lim _{t \rightarrow \infty} V_{i}=0$, and thus, $\lim _{t \rightarrow \infty} \mid y_{i}$ $y_{i, r} \mid=0$. The arguments for proving the boundness of closedloop signals are exactly the same as those in the proof of Theorem 4.

Theorem 6: Under Assumptions A1 and A2, assuming that $p=m$, if $u=u_{\mathrm{tr}}$ defined in (11) is applied to (1), then the following statements hold.

1) $\lim _{t \rightarrow \infty}\left|y_{i}-y_{i, r}\right|=0$ for any $1 \leq i \leq m$.

2) All closed-loop signals are bounded.

Proof: Because $p=m$ and according to Assumption A1, one has $G_{\mathrm{tr}}=G$, and thus, $u_{\mathrm{tr}}=u$. Following the arguments in the proof of Theorem 4 , for any $1 \leq i \leq m$, one can reach

$$
\dot{V}_{i} \leq-\sum_{j=1}^{r_{i}} c_{i, j} \xi_{i, j}^{2} .
$$

This implies that $\lim _{t \rightarrow \infty} V_{i}=0$, and thus, $\lim _{t \rightarrow \infty} \mid y_{i}$ $y_{i, r} \mid=0$, and also that $\xi_{i, j}, 1 \leq j \leq r_{i}$, and, thus, $q_{i, j}, 1 \leq$ $j \leq r_{i}$, are bounded. Because $q_{i, j}, 1 \leq j \leq r_{i}, 1 \leq i \leq m$, are bounded, $x$ is bounded. Using Assumptions A1 and A2 and the definition of $u_{\mathrm{tr}}$, it is easy to see that $u$ is bounded. The theorem is thus proved.

Remark 4: Note that for $m=p$, Assumptions A3 and A4 are not needed, and much stronger results are obtained than the case when $m<p$. If $m>p$ and $\operatorname{rank}(G(x)) \geq p$, one can always 
choose $p$ control inputs in $u$ to form $u_{p}$. By letting the other inputs to be zero, it is easy to see that the problem can be dealt with as in the case when $p=m$.

\section{Output Feedback Controller}

Note that in the design of $u_{\mathrm{tr}}$ and $u_{\mathrm{sta}}$, it is assumed that $x=$ $\left(q_{1,1} \cdots q_{1, r_{1}} \cdots q_{p, 1} \cdots q_{p, r_{p}}\right)^{T}$ is available. However, in this paper, only the outputs, i.e., $y_{1}=q_{1,1}, y_{2}=q_{2,1}, \ldots, y_{p}=$ $q_{p, 1}$ are measured. Therefore, the controller design cannot be directly implemented.

According to (1), it is easy to see that

$$
q_{i, j+1}=y_{i}^{(j)}, \quad 1 \leq i \leq p ; \quad 1 \leq j \leq r_{i}-1
$$

where $f^{(j)}$ is the $j$ th-order derivative of $f$.

This implies that

$$
x=\left(\begin{array}{lllllll}
y_{1} \dot{y}_{1} & \cdots & y_{1}^{\left(r_{1}-1\right)} & \cdots & y_{p} \dot{y}_{p} & \cdots & y_{p}^{\left(r_{p}-1\right)}
\end{array}\right)^{T} .
$$

Because the derivatives of the outputs are not measured, they have to be estimated in order to implement $u_{\text {tr }}$ and $u_{\text {sta. }}$. For this, the HOSMDs presented in Section III will be used to estimate the derivatives of the outputs. For $y_{i}$, design an $r_{i}$ thorder sliding mode differentiator of the form (2), and denote the estimates of $y_{i}$ and the derivatives of $y_{i}$ as $z_{i, 0}, z_{i, 1}, \ldots, z_{i, r_{i}-1}$. Then, one obtains an estimate of $x$ as

$$
\hat{x}=\left(\begin{array}{lllllll}
y_{1} z_{1,1} & \cdots & z_{1, r_{1}-1} & \cdots & y_{p} z_{p, 1} & \cdots & z_{p, r_{p}-1}
\end{array}\right)^{T} .
$$

Replacing $x$ with $\hat{x}$, an output feedback controller using HOSMDs is given as

$$
u=u_{\mathrm{tr}}+u_{\mathrm{sta}}
$$

where $u_{\mathrm{tr}}$ and $u_{\mathrm{sta}}$ are designed as follows:

$$
\begin{aligned}
u_{\mathrm{tr}} & =G_{\mathrm{tr}}^{-1}(\hat{x})\left(U_{\mathrm{tr}}(\hat{x})-F_{\mathrm{tr}}(\hat{x})\right) \\
u_{\mathrm{sta}} & =\left(\left(u_{p-m}\right)^{T} 0\right)^{T}
\end{aligned}
$$

where $u_{p-m}=\left(\operatorname{sat}_{M}\left(\bar{u}_{m+1}\right) \cdots \operatorname{sat}_{M}\left(\bar{u}_{p}\right)\right)^{T}$ with $\bar{u}_{p-m}=$ $\bar{G}_{\mathrm{tr}, 1}^{-1}(\hat{x}) \bar{U}_{\mathrm{tr}}(\hat{x})$, and the elements in $U_{\mathrm{tr}}(x)$ and $\bar{U}_{\mathrm{tr}}(x)$ are replaced by $U_{1}(\hat{x}), \ldots, U_{p}(\hat{x})$ and

$$
\begin{aligned}
U_{i}(\hat{x})= & -\left(c_{i, r_{i}}+1\right) \xi_{i, r_{i}}-\xi_{i, r_{i}-1}+\sum_{j=1}^{r_{i}-1} \frac{\partial \alpha_{i, r_{i}-1}}{\partial z_{i, j-1}} z_{i, j} \\
& +\sum_{j=0}^{r_{i}-1} \frac{\partial \alpha_{i, r_{i}-1}}{\partial y_{i, r}^{(j)}} y_{i, r}^{(j+1)} \\
\xi_{i, 1}= & q_{i, 1}-y_{i, r} \\
\alpha_{i, 1}= & -c_{i, 1} \xi_{i, 1}+\dot{y}_{i, r} \\
\xi_{i, k}= & z_{i, k-1}-\alpha_{i, k-1}, \quad 2 \leq k \leq r_{i} \\
\alpha_{i, k}= & -c_{i, k} \xi_{i, k}-\xi_{i, k-1}+\sum_{j=1}^{k-1} \frac{\partial \alpha_{i, k-1}}{\partial z_{i, j-1}} z_{i, j} \\
& +\sum_{j=0}^{k-1} \frac{\partial \alpha_{i, k-1}}{\partial y_{i, r}^{(j)}} y_{i, r}^{(j+1)}, \quad 2 \leq k \leq r_{i}-1
\end{aligned}
$$

where $z_{i, 0}=q_{i, 1}$.
In the remainder of this section, the output tracking error and closed-loop stability using the output feedback controller will be analyzed. To this end, define $\sigma_{i, 0}=y_{i}-z_{i, 0}, \sigma_{i, 1}=$ $\dot{y}_{i}-z_{i, 1}, \ldots, \sigma_{i, r_{i}-1}=y_{i}^{\left(r_{i}-1\right)}-z_{i, r_{i}-1}, 1 \leq i \leq p$, and $\sigma=$ $\left(\begin{array}{lllllll}\sigma_{1,0} & \cdots & \sigma_{1, r_{1}-1} & \cdots & \sigma_{p, 0} & \cdots & \sigma_{1, r_{p}-1}\end{array}\right)^{T}$, and denote $\Omega=\{x\|x\| \leq \delta\}$ and $\Psi=\{\sigma\|\sigma\| \leq \delta\}$ for any $\delta>0$.

The main stability result is presented in Theorem 7 .

Theorem 7: Given Assumptions A1-A4, and assuming that there is no measurement noise and $c_{i, j}, 1 \leq i \leq p, 1 \leq j \leq$ $r_{i}$, are chosen to be large enough, if the controller given in (27)-(30) is applied to (1), then, for any $x(0) \times \sigma(0) \in \Omega \times \Psi$, the following statements hold.

1) The output tracking errors, i.e., $\left|y_{i}-y_{i, r}\right|$, will enter a small neighborhood of zero for any $1 \leq i \leq m$, and moreover, $\lim _{t \rightarrow \infty}\left|y_{i}-y_{i, r}\right|=0$ for any $1 \leq i \leq m$ if $\lim _{t \rightarrow \infty} u_{\text {sta }}=0$.

2) All closed-loop signals are bounded.

Proof: Define $\bar{\Omega}=\left\{\bar{x}^{1} \mid V_{1} \leq\left(\bar{N}_{1} / 2 c_{1, \min }\right), \ldots, V_{m} \leq\right.$ $\left.\left(\bar{N}_{m} / 2 c_{m, \min }\right)\right\}$.

Now, choose $\delta_{2}>\delta_{1}>\delta$, and define for $i=1,2, \Omega_{i}=$ $\left\{x\|x\| \leq \delta_{i}\right\}$ and $\Psi_{i}=\left\{\sigma\|\sigma\| \leq \delta_{i}\right\}$ such that $\Omega \times \Psi \subset \Omega_{1} \times$ $\Psi_{1} \subset \Omega_{2} \times \Psi_{2}$.

With the controller given in (27)-(30) being applied to (1) under Assumptions A1-A4, there exists a positive constant $M_{\delta}$ such that for any $(x, \sigma) \in \Omega \times \Psi,\|\dot{x}\| \leq M_{\delta}$. Similarly, there exist positive constants $M_{\delta_{1}}$ and $M_{\delta_{2}}$ such that for any $(x, \sigma) \in$ $\Omega_{i} \times \Psi_{i},\|\dot{x}\| \leq M_{\delta_{i}}, i=1,2$.

For any $x(0) \times \sigma(0) \in \Omega \times \Psi$, because $\|\dot{x}\| \leq M_{\delta_{1}}$ for any $(x, \sigma) \in \Omega_{1} \times \Psi_{1}$, there exists $T_{1}>0$ such that for any $t \in$ $\left[0, T_{1}\right],\|x\| \leq \delta_{1}$.

Because there is no measurement noise, according to Theorem 1, exact derivative reconstruction can be achieved after some transient time. Moreover, according to [9], the transient time can be made arbitrarily small by choosing the design constants in the differentiators to be sufficiently large. Therefore, by choosing the design constants in the differentiators to be large enough (which is possible because $\|\dot{x}\| \leq M_{\delta_{1}}$ within $\left[0, T_{1}\right]$ ), exact derivative reconstruction can be achieved within $\left[0, T_{1}\right]$.

Using (18), it can be shown that $\bar{\Omega}$ is a positive invariant attractive set of $\bar{x}^{1}$. Therefore, $\bar{x}^{1}(t)$ is bounded. This, together with Assumption 4, guarantees that $\bar{x}^{2}(t)$ is bounded. Hence, $\|\dot{x}\|$ is bounded, and there exists $M_{\delta_{2}}$ such that $\|\dot{x}\| \leq M_{\delta_{2}}$ for all $t \geq 0$. Therefore, by choosing the design constants in the differentiators to be sufficiently large (according to $M_{\delta_{2}}$ ), exact derivative reconstruction can be achieved for all $t \geq T_{1}$.

Because $\hat{x}=x$ for all $t \geq T_{1}$, the remaining part of the conclusions can be proved the same way as in the proof of Theorem 4.

Remark 5: Using similar arguments as in the proof of Theorem 7, it is possible to give results corresponding to those provided in Theorems 5 and 6.

Remark 6: It should be pointed out that all the results are proved under the assumption that exact derivative reconstruction can be achieved after some transient time. In real applications, exact derivative reconstruction is not achievable because of noise and sampling rate restrictions. In the presence 


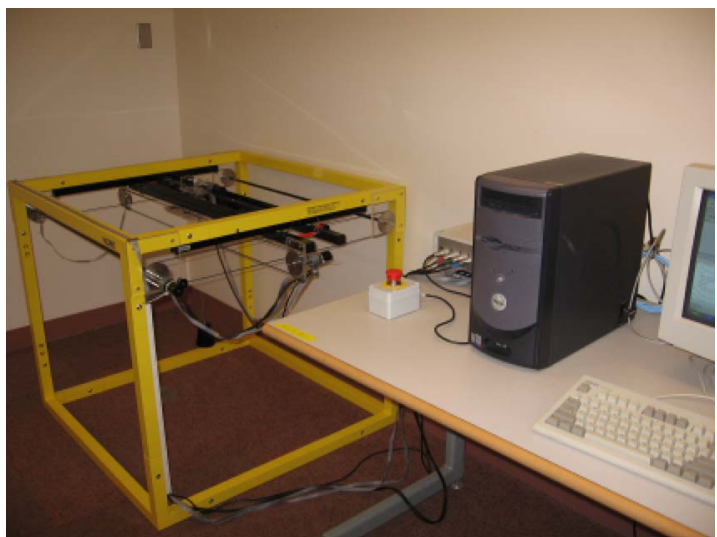

Fig. 1. Experimental setup. A laboratory-scale 3-D crane system.

of noise and sampling rate restrictions, the derivative estimation error can be taken care of by choosing the design constants $c_{i, j}, 1 \leq i \leq p, 1 \leq j \leq r_{i}$, to be large enough because of the virtue of the backstepping approach. More discussions will be provided on this point using experimental results.

\section{Applications to a Laboratory-SCAle 3-D CRANE: EXPERIMENTAL RESULTS}

Overhead cranes are widely used for material transportation in many industrial applications [12]-[14], which motivates us to choose a laboratory-scale 3-D crane as an experimental example. In this section, a brief introduction to a laboratory-scale 3-D crane and its nonlinear mathematical model is first presented. Then, an output feedback controller using an HOSMD is designed for this system by applying the controller design proposed in Section IV. After that, the designed controller is tested, and experimental results are provided. Finally, some discussions based on the experimental results are made on several issues related to the controller design.

\section{A. A Laboratory-Scale 3-D Crane System and Its Nonlinear Mathematical Model}

In this section, a brief introduction of the 3-D crane system and its nonlinear mathematical model is presented. The experimental setup of the 3-D crane system provided by the InTeCo Ltd is shown in Fig. 1.

In the experimental setup shown in Fig. 1, the 3-D crane consists of a construction frame, a rail attached to the frame that moves along the frame, a cart that moves on the rail, and a payload that is shifted up and down.

The mathematical model used for the output feedback controller design takes the following form:

$$
\begin{aligned}
& \dot{x}_{1}=x_{2} \\
& \dot{x}_{2}=\Phi_{1}+\mu_{1} \cos \left(x_{5}\right) \Phi_{3} \\
& \dot{x}_{3}=x_{4} \\
& \dot{x}_{4}=\Phi_{2}+\mu_{2} \sin \left(x_{5}\right) \sin \left(x_{7}\right) \Phi_{3} \\
& \dot{x}_{5}=x_{6}
\end{aligned}
$$

\begin{tabular}{|c|c|}
\hline Variable & Definition \\
\hline$x_{1}$ & $\begin{array}{l}\text { the position of the cart } \\
\text { from the center of the rail }\end{array}$ \\
\hline$x_{2}$ & the velocity along $x_{1}$ \\
\hline$x_{3}$ & $\begin{array}{l}\text { the position of the rail from } \\
\text { the center of the construction frame }\end{array}$ \\
\hline$x_{4}$ & the velocity along $x_{3}$ \\
\hline$x_{5}$ & $\begin{array}{c}\text { the acute angle between the lift-line } \\
\text { of the payload and the rail }\end{array}$ \\
\hline$x_{6}$ & the angular velocity related to $x_{5}$ \\
\hline$x_{7}$ & $\begin{array}{l}\text { the acute angle between the lift-line } \\
\text { of the payload and the vertical line }\end{array}$ \\
\hline$x_{8}$ & the angular velocity related to $x_{7}$ \\
\hline$x_{9}$ & the length of the lift-line \\
\hline$x_{10}$ & the changing rate of the length of the lift-line \\
\hline
\end{tabular}

TABLE I

Definition of the State Variables

$$
\begin{aligned}
\dot{x}_{6}= & \left(\sin \left(x_{5}\right) \Phi_{1}-\cos \left(x_{5}\right) \sin \left(x_{7}\right) \Phi_{2}\right) / x_{9} \\
& +\left(\left(\mu_{1}-\mu_{2} \sin ^{2}\left(x_{7}\right)\right) \sin \left(x_{5}\right) \cos \left(x_{5}\right) \Phi_{3}+\Psi_{5}\right) / x_{9} \\
\dot{x}_{7}= & x_{8} \\
\dot{x}_{8}= & -\left(\cos \left(x_{7}\right) \Phi_{2}+\Psi_{6}\right) /\left(\sin \left(x_{5}\right) x_{9}\right) \\
& -\mu_{2} \sin \left(x_{5}\right) \cos \left(x_{7}\right) \sin \left(x_{7}\right) \Phi_{3} /\left(\sin \left(x_{5}\right) x_{9}\right) \\
\dot{x}_{9}= & x_{10} \\
\dot{x}_{10}= & -\cos \left(x_{5}\right) \Phi_{1}-\sin \left(x_{5}\right) \sin \left(x_{7}\right) \Phi_{2}+\Psi_{7} \\
& -\left(1+\mu_{1} \cos ^{2}\left(x_{5}\right)+\mu_{2} \sin ^{2}\left(x_{5}\right) \sin ^{2}\left(x_{7}\right)\right) \Phi_{3} \\
y_{i}= & x_{2(i-1)+1}, \quad 1 \leq i \leq 5
\end{aligned}
$$

where $\Phi_{i}, i=1,2,3$, are defined as

$$
\begin{aligned}
& \Phi_{1}=k_{1} u_{1}-T_{1} x_{2}-T_{s y} \operatorname{sign}\left(x_{2}\right) \\
& \Phi_{2}=k_{2} u_{2}-T_{2} x_{4}-T_{s x} \operatorname{sign}\left(x_{4}\right) \\
& \Phi_{3}=k_{3} u_{3}+T_{3} x_{10}+T_{s z} \operatorname{sign}\left(x_{10}\right)
\end{aligned}
$$

and $\Psi_{i}, i=5,6,7$, are defined as

$$
\begin{aligned}
& \Psi_{5}=\sin \left(x_{5}\right) \cos \left(x_{5}\right) x_{8}^{2} x_{9}+g \cos \left(x_{5}\right) \cos \left(x_{7}\right)-2 x_{6} x_{10} \\
& \Psi_{6}=-g \sin \left(x_{7}\right)-2 x_{6} x_{8} x_{9} \cos \left(x_{5}\right)-2 x_{8} x_{10} \sin \left(x_{5}\right) \\
& \Psi_{7}=x_{8}^{2} x_{9} \sin ^{2}\left(x_{5}\right)+g \sin \left(x_{5}\right) \cos \left(x_{7}\right)+x_{6}^{2} x_{9}
\end{aligned}
$$

The definition of the variables in the above model are provided in Tables I and II.

All parameters in the model given in (31)-(33) are listed as follows:

$$
\begin{aligned}
& \mu_{1}=0.4156 \\
& \mu_{2}=0.1431 \\
& T_{s y}=6.4935 \quad T_{s x}=1.4903 \\
& k_{1}=49.8636 \quad k_{2}=16.0336 \\
& T_{1}=11.5242 \quad T_{2}=26.3263 \quad T_{3}=217.3535 .
\end{aligned}
$$


TABLE II

DEFINITION OF OTHER VARIABLES

\begin{tabular}{|c|c|}
\hline Variable & Definition \\
\hline$u_{1}$ & $\frac{F_{y}}{m_{w}}$ \\
$u_{3}$ & $\frac{F_{x}}{\left(m_{w}+m_{c}\right)}$ \\
$\mu_{1}$ & $\frac{F_{R}}{m_{c}}$ \\
$\mu_{2}$ & $\frac{m_{c}}{m_{w}}$ \\
$m_{c}$ & mass of the payload \\
$m_{w}$ & mass of the cart \\
$m_{s}$ & mass of the moving rail \\
$F_{x}$ & forces driving the rail with cart \\
$F_{y}$ & forces driving the cart along the rail \\
$F_{R}$ & forces controlling the length of the lift-line \\
$T_{1}, T_{2}, T_{3}$ & friction related coefficients \\
$T_{s x}, T_{s y}, T_{s z}$ & friction related coefficients \\
\hline
\end{tabular}

Denote $x=\left(\begin{array}{lllll}q_{1,1} & q_{1,2} & \cdots & q_{5,1} & q_{5,2}\end{array}\right)^{T} \in R^{10}$. Then, one has

$$
\begin{array}{ll}
q_{1,1}=x_{1} & q_{1,2}=x_{2} \\
q_{2,1}=x_{3} & q_{2,2}=x_{4} \\
q_{3,1}=x_{5} & q_{3,2}=x_{6} \\
q_{4,1}=x_{7} & q_{4,2}=x_{8} \\
q_{5,1}=x_{9} & q_{5,2}=x_{10} .
\end{array}
$$

Hence, the model given in (31)-(33) can be rewritten in the form of (1) as

$$
\begin{aligned}
\dot{q}_{i, 1} & =q_{i, 2} \\
\dot{q}_{i, 2} & =f_{i}(x)+G_{i}(x) u \\
y_{i} & =q_{i, 1}, \quad 1 \leq i \leq 5
\end{aligned}
$$

where

$$
\begin{aligned}
f_{1}= & -T_{1} x_{2}-T_{s y} \operatorname{sign}\left(x_{2}\right) \\
& +\mu_{1} \cos \left(x_{5}\right)\left(T_{3} x_{10}+T_{s z} \operatorname{sign}\left(x_{10}\right)\right) \\
f_{2}= & -T_{2} x_{4}-T_{s x} \operatorname{sign}\left(x_{4}\right)+\mu_{2} T_{3} \sin \left(x_{5}\right) \sin \left(x_{7}\right) x_{10} \\
& +\mu_{2} T_{s z} \sin \left(x_{5}\right) \sin \left(x_{7}\right) \operatorname{sign}\left(x_{10}\right) \\
f_{3}= & -\sin \left(x_{5}\right)\left(T_{1} x_{2}+T_{s y} \operatorname{sign}\left(x_{2}\right)\right) / x_{9} \\
& +\cos \left(x_{5}\right) \sin \left(x_{7}\right)\left(T_{2} x_{4}+T_{s x} \operatorname{sign}\left(x_{4}\right)\right) / x_{9} \\
& +\frac{\left(\mu_{1}-\mu_{2} \sin ^{2}\left(x_{7}\right)\right) \sin \left(x_{5}\right) \cos \left(x_{5}\right) T_{3} x_{10}}{x_{9}} \\
& +\frac{\left(\mu_{1}-\mu_{2} \sin ^{2}\left(x_{7}\right)\right) \sin \left(x_{5}\right) \cos \left(x_{5}\right) T_{s z} \operatorname{sign}\left(x_{10}\right)}{x_{9}} \\
& +\Psi_{5} / x_{9}
\end{aligned}
$$

$$
\begin{aligned}
f_{4}= & \cos \left(x_{7}\left(T_{2} x_{4}+T_{s x} \operatorname{sign}\left(x_{4}\right)\right) /\left(x_{9} \sin \left(x_{5}\right)\right)\right. \\
& -\frac{\mu_{2} \sin \left(x_{5}\right) \cos \left(x_{7}\right) \sin \left(x_{7}\right) T_{3} x_{10}}{x_{9} \sin \left(x_{5}\right)} \\
& -\frac{\mu_{2} \sin \left(x_{5}\right) \cos \left(x_{7}\right) \sin \left(x_{7}\right) T_{s z} \operatorname{sign}\left(x_{10}\right)}{x_{9} \sin \left(x_{5}\right)} \\
& -\Psi_{6} /\left(x_{9} \sin \left(x_{5}\right)\right) \\
f_{5}= & \cos \left(x_{5}\right)\left(T_{1} x_{2}+T_{s y} \operatorname{sign}\left(x_{2}\right)\right) \\
& +\sin \left(x_{5}\right) \sin \left(x_{7}\right)\left(T_{2} x_{4}+T_{s x} \operatorname{sign}\left(x_{4}\right)\right) \\
& -\left(1+\mu_{1} \cos ^{2}\left(x_{5}\right)\right)\left(T_{3} x_{10}+T_{s z} \operatorname{sign}\left(x_{10}\right)\right) \\
& -\mu_{2} \sin ^{2}\left(x_{5}\right) \sin ^{2}\left(x_{7}\right)\left(T_{3} x_{10}+T_{s z} \operatorname{sign}\left(x_{10}\right)\right)+\Psi_{7}
\end{aligned}
$$

and $G_{i}=\left(g_{i 1}, g_{i 2}, g_{i 3}\right), 1 \leq i \leq 5$, with their elements defined as

$$
\begin{aligned}
& g_{1,1}=k_{1} \\
& g_{1,2}=0 \\
& g_{1,3}=\mu_{1} k_{3} \cos \left(x_{5}\right) \\
& g_{2,1}=0 \\
& g_{2,2}=k_{2} \\
& g_{2,3}=\mu_{2} k_{3} \sin \left(x_{5}\right) \sin \left(x_{7}\right) \\
& g_{3,1}=k_{1} \sin \left(x_{5}\right) / x_{9} \\
& g_{3,2}=-k_{2} \cos \left(x_{5}\right) \sin \left(x_{7}\right) / x_{9} \\
& g_{3,3}=\frac{k_{3} \sin \left(x_{5}\right) \cos \left(x_{5}\right)\left(\mu_{1}-\mu_{2} \sin ^{2}\left(x_{7}\right)\right)}{g_{9}} \\
& g_{4,1}=0 \\
& g_{4,2}=-k_{2} \cos \left(x_{7}\right) /\left(x_{9} \sin \left(x_{5}\right)\right) \\
& g_{4,3}=-\mu_{2} k_{3} \sin \left(x_{5}\right) \sin \left(x_{7}\right) \cos \left(x_{7}\right) /\left(x_{9} \sin \left(x_{5}\right)\right) \\
& g_{5,1}=-k_{1} \cos \left(x_{5}\right) \\
& g_{5,2}=-k_{2} \sin \left(x_{5}\right) \sin \left(x_{7}\right) \\
& g_{5,3}=k_{3}\left(-\mu_{1} \cos { }^{2}\left(x_{5}\right)-\mu_{2} \sin ^{2}\left(x_{5}\right) \sin ^{2}\left(x_{7}\right)-1\right) .
\end{aligned}
$$

\section{B. Output Feedback Controller Design}

For the system given in (36)-(38), it is easy to see that $m=3$ and $p=5$, and that $m<p$. The control objective is to design an output feedback controller to make $y_{1}, y_{2}$, and $y_{5}$ track their desired reference signals and ensure that the signals in the closed-loop system are bounded. For this problem, one has $m_{\mu}=m=3$.

According to Section IV, $G_{\mathrm{tr}}=\left(G_{1} G_{2} G_{5}\right)^{T}, F_{\mathrm{tr}}(x)=$ $\left(\begin{array}{lll}f_{1} & f_{2} & f_{5}\end{array}\right)^{T}, \bar{G}_{\mathrm{tr}}(x)=\left(\begin{array}{ll}G_{3} & G_{4}\end{array}\right)^{T}, \bar{F}_{\mathrm{tr}}(x)=\left(\begin{array}{ll}f_{3} & f_{4}\end{array}\right)^{T}$, and $\bar{G}_{\mathrm{tr}, 1}(x)$ consists of the first two columns of $\bar{G}_{\mathrm{tr}}(x)$.

Following the output feedback control design procedure in Section IV, an output feedback controller using HOSMDs can be designed as

$$
u=u_{\mathrm{tr}}+u_{\mathrm{sta}}
$$



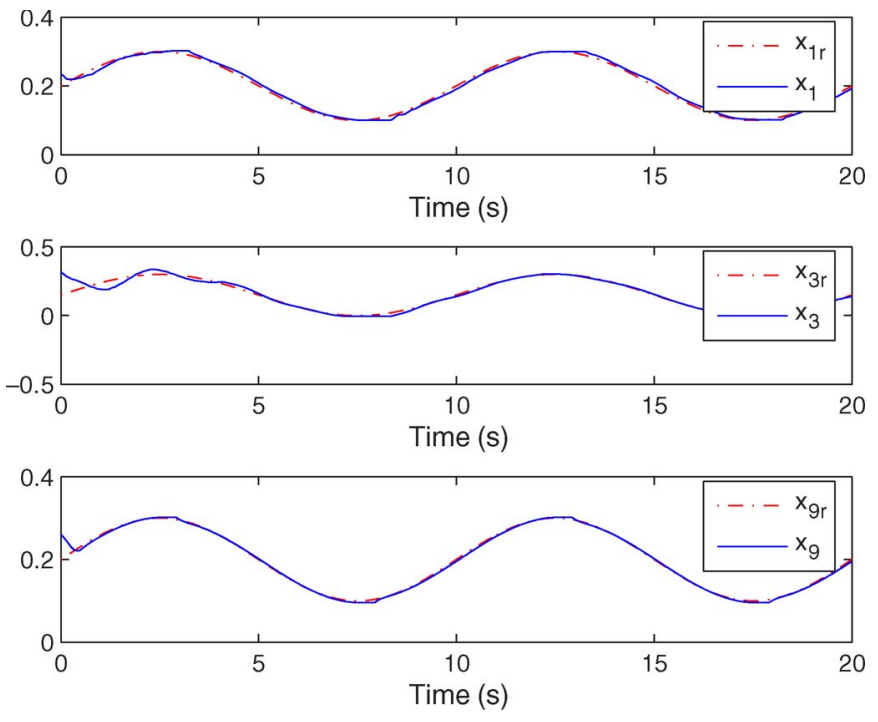

Fig. 2. Tracking effect of the chosen outputs.

where $u_{\mathrm{tr}}$ and $u_{\mathrm{sta}}$ are designed as follows:

$$
\begin{aligned}
u_{\mathrm{tr}} & =G_{\mathrm{tr}}^{-1}(\hat{x})\left(U_{\mathrm{tr}}(\hat{x})-F_{\mathrm{tr}}(\hat{x})\right) \\
u_{\mathrm{sta}} & =\left(u_{2}^{T} 0\right)^{T}
\end{aligned}
$$

where $u_{2}=\left(\operatorname{sat}_{M}\left(\bar{u}_{4}\right) \quad \operatorname{sat}_{M}\left(\bar{u}_{5}\right)\right)^{T}$, with $\bar{u}_{2}=\left(\bar{u}_{4} \bar{u}_{5}\right)^{T}=$ $\bar{G}_{\mathrm{tr}, 1}^{-1}(\hat{x}) \bar{U}_{\mathrm{tr}}(\hat{x})$, and $U_{\mathrm{tr}}(\hat{x})=\left(\begin{array}{lll}U_{1}(\hat{x}) & U_{2}(\hat{x}) & U_{5}(\hat{x})\end{array}\right)^{T}$ and

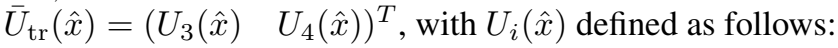

$$
\begin{aligned}
U_{i}(\hat{x}) & =-c_{i, 2} \xi_{i, 2}-\xi_{i, 1}-c_{i, 1} z_{i, 1}+c_{i, 1} \dot{y}_{i, r}+\ddot{y}_{i, r} \\
\xi_{i, 1} & =q_{i, 1}-y_{i, r} \\
\alpha_{i, 1} & =-c_{i, 1} \xi_{i, 1}+\dot{y}_{i, r} \\
\xi_{i, 2} & =z_{i, 1}-\alpha_{i, 1} .
\end{aligned}
$$

To provide the estimates for $\dot{y}_{i}, 1 \leq i \leq 5$, for each $1 \leq i \leq$ 5 , a second-order sliding-mode differentiator with the following form is used:

$$
\begin{aligned}
& \dot{z}_{i, 0}=v_{i, 0} \\
& v_{i, 0}=-\lambda_{0}\left|z_{i, 0}-y_{i}\right|^{2 / 3} \operatorname{sign}\left(z_{i, 0}-y_{i}\right)+z_{i, 1} \\
& \dot{z}_{i, 1}=v_{i, 1} \\
& v_{i, 1}=-\lambda_{1}\left|z_{i, 1}-v_{i, 0}\right|^{1 / 2} \operatorname{sign}\left(z_{i, 1}-v_{i, 0}\right)+z_{i, 2} \\
& \dot{z}_{i, 2}=-\lambda_{2} \operatorname{sign}\left(z_{i, 2}-v_{i, 1}\right)
\end{aligned}
$$

where $z_{i, 1}$ provides the estimate for $\dot{y}_{i}$.

Remark 7: Since only the first-order derivatives need to be estimated, first-order differentiators are also able to provide estimation for those first-order derivatives. The reason for using the second-order differentiators is that they may provide a slightly better estimation accuracy in the presence of measurement noises and the discrete sampling (Theorems 2 and 3).
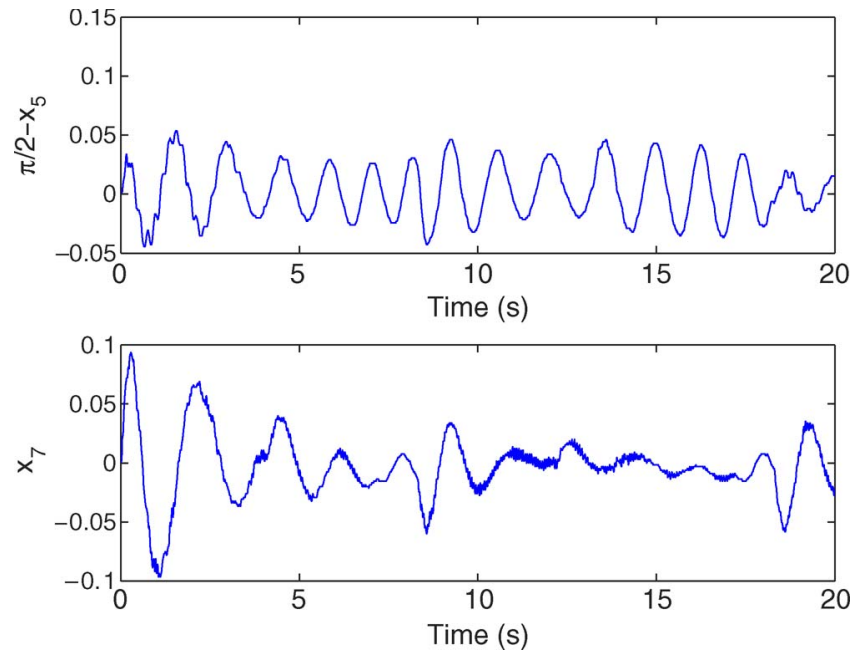

Fig. 3. Stabilization effect of the angles.
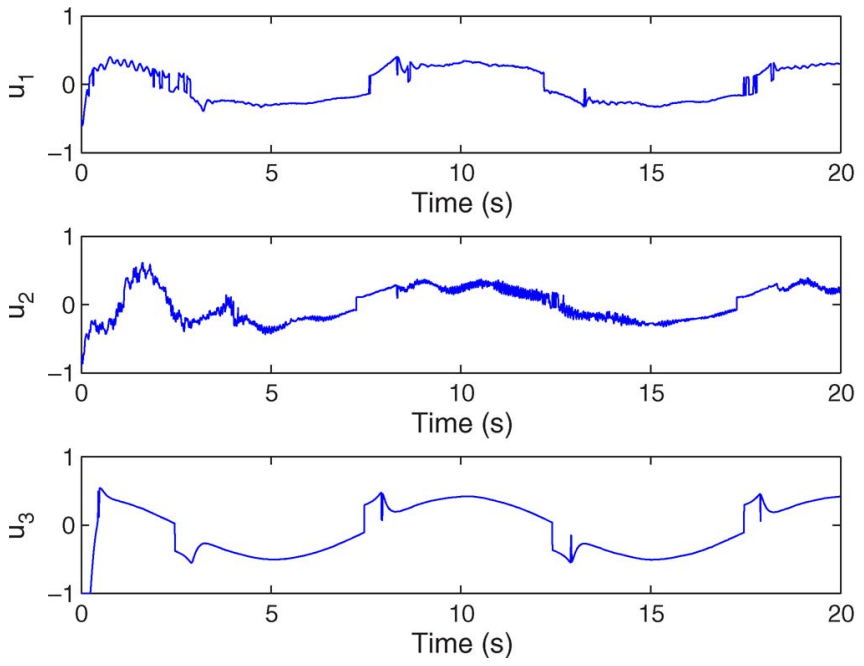

Fig. 4. Control effort.

\section{Experimental Results on the 3-D Crane System}

In order to test the applicability of the output feedback controller proposed in this paper to practical industrial systems, experiments are carried out on the 3-D crane system.

In all experiments, the design constants for second-order sliding mode differentiators are chosen as $\lambda_{0}=\lambda_{1}=20$ and $\lambda_{2}=30$, and the design constants in the controller are chosen as $c_{1,1}=c_{1,2}=30, c_{2,1}=c_{2,2}=10, c_{3,1}=c_{3,2}=15$, $c_{4,1}=c_{4,2}=15$, and $c_{5,1}=c_{5,2}=150$, except in one particular experiment, where $c_{1,1}=c_{1,2}=9, c_{2,1}=c_{2,2}=3, c_{3,1}=$ $c_{3,2}=3, c_{4,1}=c_{4,2}=3$, and $c_{5,1}=c_{5,2}=45$. The sampling period is chosen as $T_{s}=0.01 \mathrm{~s}$, except in one experiment, where $T_{s}=0.002 \mathrm{~s}$.

The tracking reference signals are chosen as $y_{1, r}=y_{5, r}=$ $0.2+0.1 \sin (0.2 \pi t), \quad y_{2, r}=0.15+0.15 \sin (0.2 \pi t), \quad y_{3, r}=$ $\pi / 2$, and $y_{4, r}=0$.

Four experiments were performed.

The first experiment illustrates successful application of the controller given in (39)-(43) to the 3-D crane system. The results are presented in Figs. 2-4. 

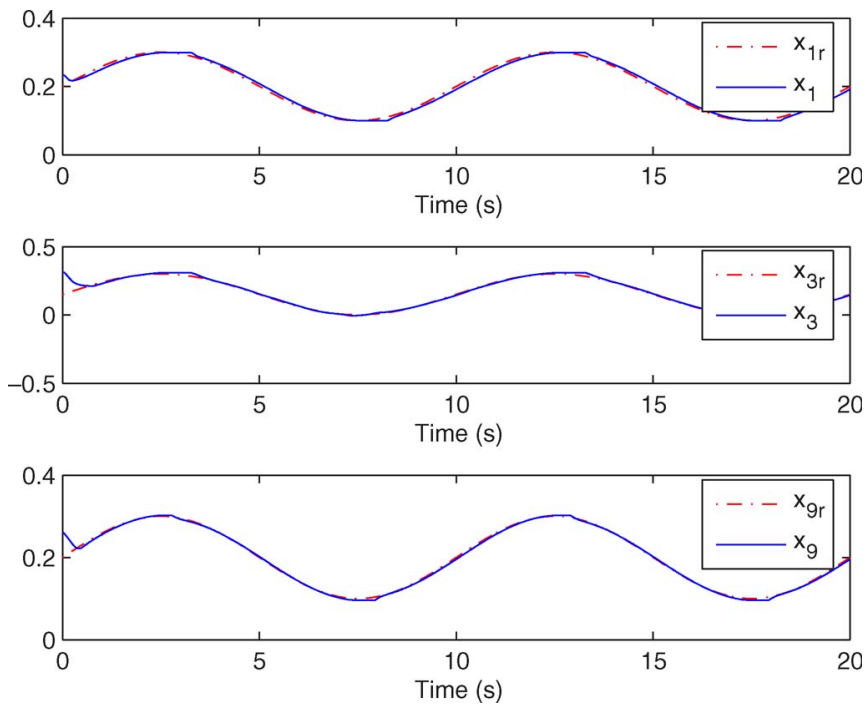

Fig. 5. Tracking effect of the chosen outputs: $u_{\text {sta }}=0$.
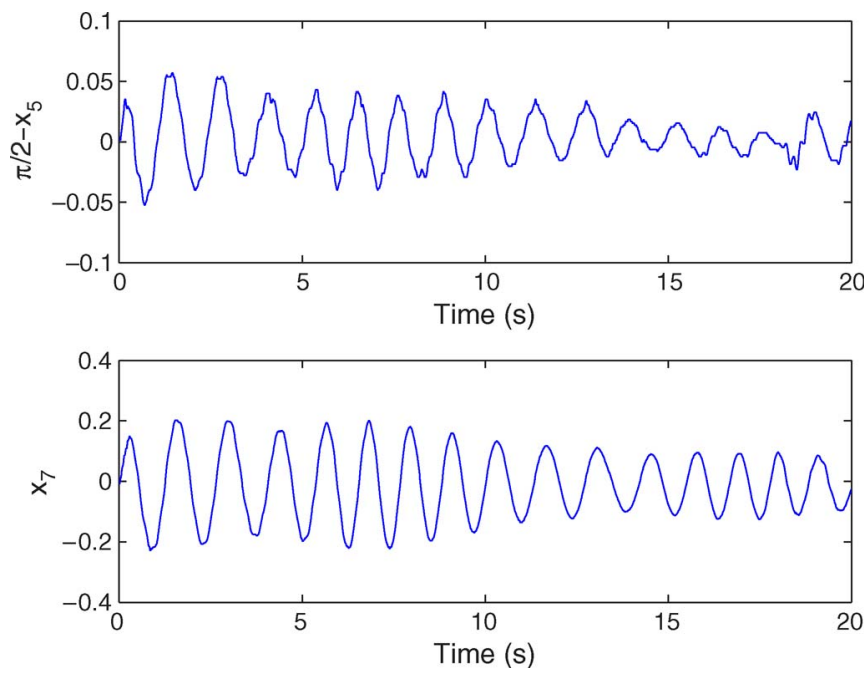

Fig. 6. Stabilization effect of the angles: $u_{\text {sta }}=0$.

The second experiment was carried out to illustrate what happens if $u_{\text {sta }}=0$, and the results are shown in Figs. 5-7.

The third experiment was intended to show the effect of the design constants in the proposed controller, and the results are provided in Figs. 8-10 for the case where $c_{1,1}=c_{1,2}=9$, $c_{2,1}=c_{2,2}=3, c_{3,1}=c_{3,2}=3, c_{4,1}=c_{4,2}=3$, and $c_{5,1}=$ $c_{5,2}=45$.

The last experiment shows the effect of sampling rate, and the results are illustrated in Figs. 11-14.

\section{Discussions Based on the Experimental Results}

The results presented in Figs. 2-4 show that the output tracking performance and angle stabilization performance are very satisfactory.

If $u_{\text {sta }}=0$, a good output tracking performance can also be achieved as can be seen in Fig. 5 with similar control effort (see Fig. 4). However, the angle $x_{7}$ in Fig. 6 is much larger than that in Fig. 3, which is not desirable. This result implies that the introduction of the design component $u_{\mathrm{sta}}$ can be beneficial.
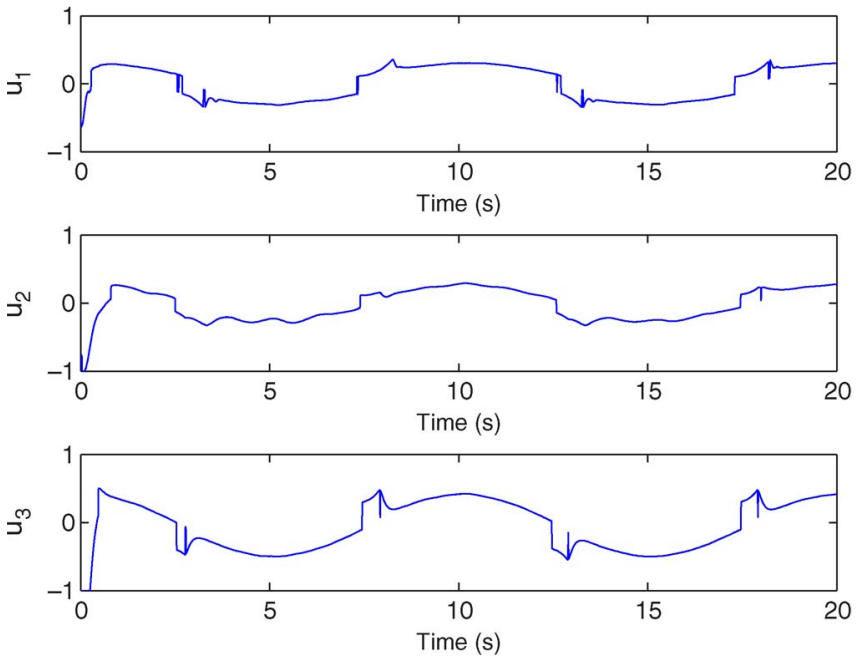

Fig. 7. Control effort: $u_{\text {sta }}=0$.
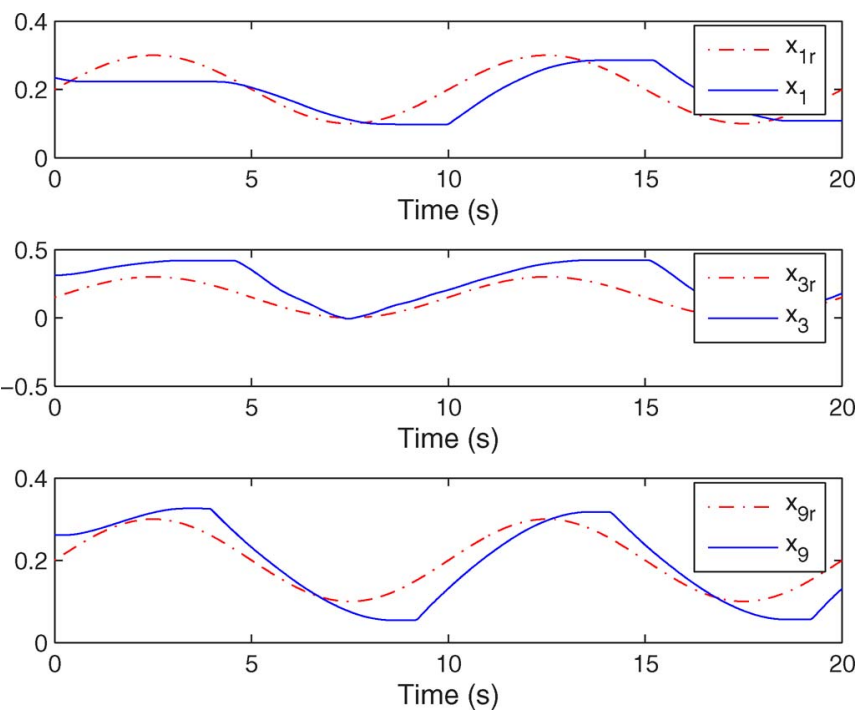

Fig. 8. Tracking effect of the chosen outputs: smaller design constants.

Because HOSMDs could not produce derivative estimation with enough accuracy according to Theorem 3 for $T_{s}=0.01 \mathrm{~s}$, those derivative estimation errors have to be taken care of through the design of the controller constants before one can achieve a satisfactory control performance in practical applications. For the case that $c_{1,1}=c_{1,2}=9, c_{2,1}=c_{2,2}=3, c_{3,1}=$ $c_{3,2}=3, c_{4,1}=c_{4,2}=3$, and $c_{5,1}=c_{5,2}=45$, although the control effort in Fig. 10 is smaller, the results in Figs. 8 and 9 show a very poor control performance. Therefore, the controller design constants should be chosen suitably large to obtain a satisfactory control performance.

Again, according to Theorem 3, one should be able to achieve a better performance by sampling faster. This conclusion is confirmed by the last experiment, with $T_{s}=0.002 \mathrm{~s}$. The results in Figs. 11 and 12 show a performance better than those shown in Figs. 2 and 3 with similar control effort (see Figs. 4 and 13). This point can be seen more clearly in the comparison result presented in Fig. 14.

In summary, experimental results show that the output feedback controller proposed in this paper has achieved a satisfactory performance when applied to a laboratory-scale 3-D 

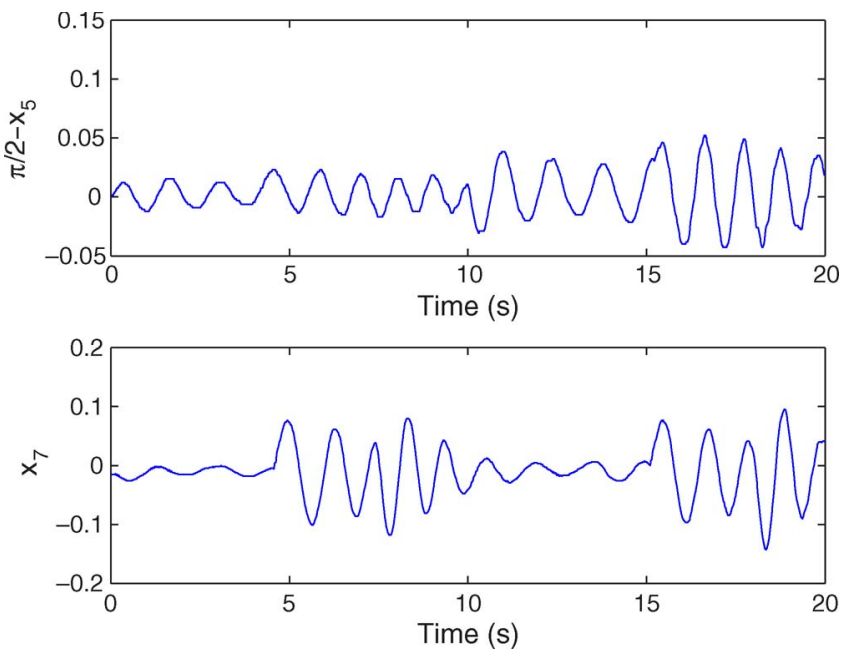

Fig. 9. Stabilization effect of the angles: smaller design constants.
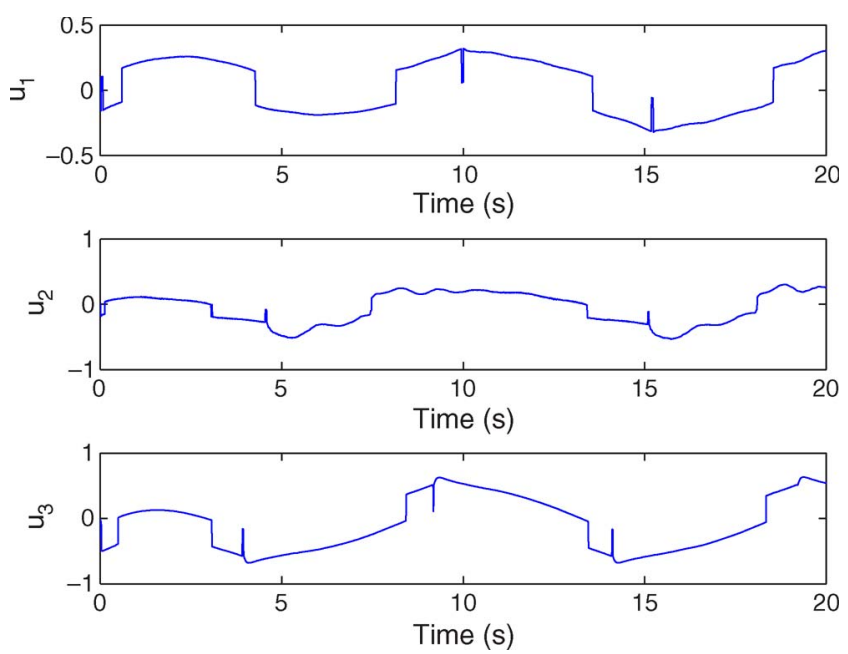

Fig. 10. Control effort: smaller design constants.
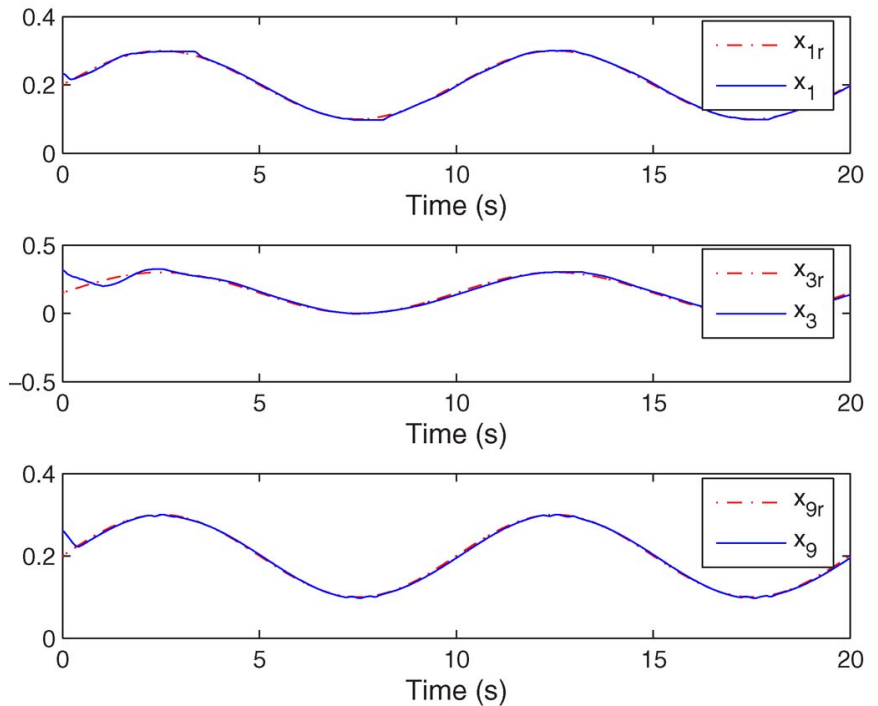

Fig. 11. Tracking effect of the chosen outputs: faster sampling.

crane system. They also show that the two design component controller structures actually can improve control performance. The experimental results also reveal that the controller design
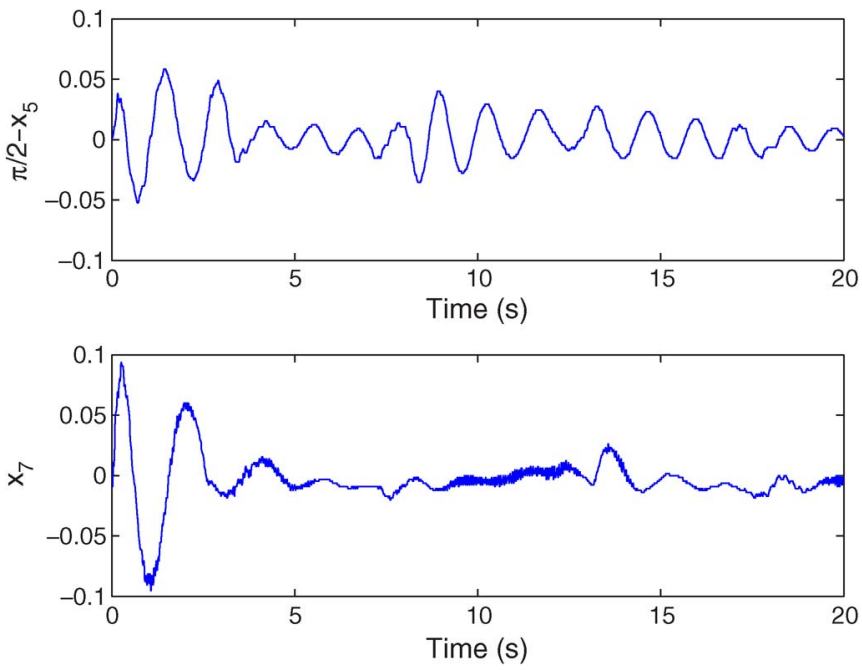

Fig. 12. Stabilization effect of the angles: faster sampling.
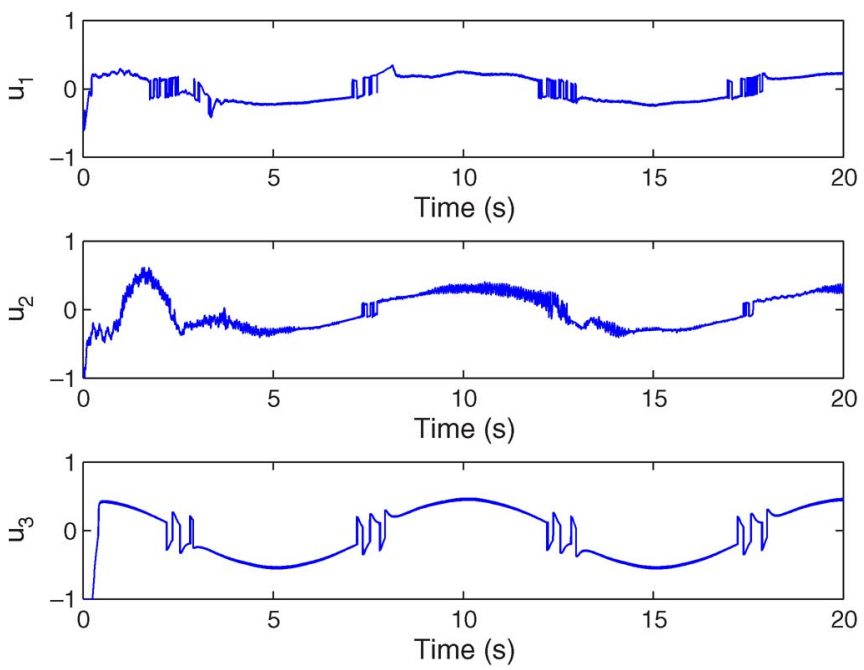

Fig. 13. Control effort: faster sampling.
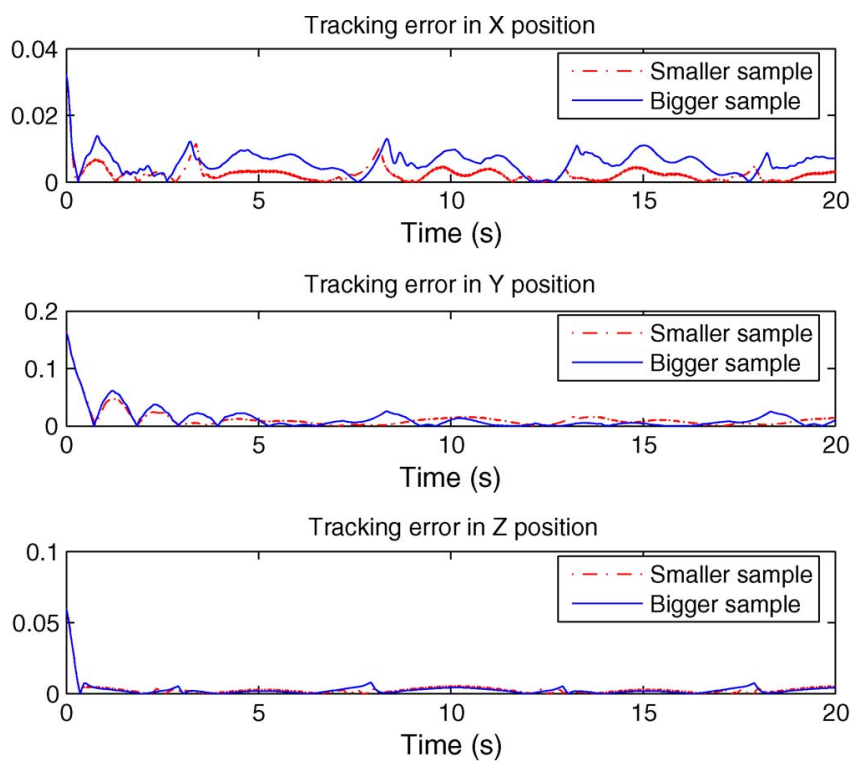

Fig. 14. Tracking error of the chosen outputs: fast sampling versus slow sampling. 
constants should be chosen to be large enough if the sampling rate is not fast enough in order to use HOSMDs in the controller design.

\section{CONCLUSION}

This paper has addressed a practical output feedback control design problem aiming to regulate only part of the outputs for a class of MIMO nonlinear systems where the number of inputs is less than that of outputs. To solve the problem, a controller structure with two design components in all or some chosen control inputs was proposed, where the two design components were designed using the backstepping approach, together with the use of the recently developed HOSMDs to estimate the derivatives of the outputs. Stability results were established for the proposed controller under certain conditions.

In order to show the practicality of the proposed output feedback controller, the controller was implemented and tested on a laboratory-scale 3-D crane system. The experimental results demonstrated that the proposed output feedback controller is able to achieve very satisfactory control performance. They have also revealed the advantage of the proposed controller structure and the effect of the controller design constants and sampling periods.

Although the experimental results confirm that a carefully designed $u_{\text {sta }}$ is helpful in the control of the damping of the swing, a strict analysis has not been performed on this yet. This is so, because the current research focused mainly on precise positioning. As such, a future research topic is to explore how good position tracking with reduced sway can be achieved.

\section{ACKNOWLEDGMENT}

The authors would like to thank the anonymous reviewers for their careful reading of the manuscript and their many questions and suggestions that improved the original draft.

\section{REFERENCES}

[1] V. I. Utkin, Sliding Modes in Control Optimization. New York: SpringerVerlag, 1992.

[2] R. A. DeCarlo, S. H. Zak, and G. H. Matthews, "Variable structure control of nonlinear multivariable systems: A tutorial," Proc. IEEE, vol. 76, no. 3, pp. 212-232, Mar. 1988

[3] J. Y. Hung, W. Gao, and J. C. Hung, "Variable structure control: A survey," IEEE Trans. Ind. Electron., vol. 40, no. 1, pp. 2-22, Feb. 1993.

[4] C. Edwards and S. K. Spurgeon, Sliding Modes Control: Theory and Applications. London, U.K.: Taylor \& Francis, 1998.

[5] H. M. Gutierrez and P. I. Ro, "Magnetic servo levitation by sliding-mode control of nonaffine systems with algebraic input invertibility," IEEE Trans. Ind. Electron., vol. 52, no. 5, pp. 1449-1455, Oct. 2005.

[6] G. Edelbaher, K. Jezernik, and E. Urlep, "Low-speed sensorless control of induction machine," IEEE Trans. Ind. Electron., vol. 53, no. 1, pp. 120129, Feb. 2006.

[7] M. Comanescu and L. Xu, "Sliding-mode MRAS speed estimators for sensorless vector control of induction machine," IEEE Trans. Ind. Electron., vol. 53, no. 1, pp. 146-153, Feb. 2006.

[8] D.-J. Kim, K.-H. Park, and Z. Bien, "Hierarchical longitudinal controller for rear-end collision avoidance," IEEE Trans. Ind. Electron., vol. 54, no. 2, pp. 805-817, Apr. 2007.

[9] A. Levant, "Higher-order sliding modes, differentiation and outputfeedback control," Int. J. Control, vol. 76, no. 9/10, pp. 924-941, Jun. 2003.

[10] G. Bartolini, A. Pisano, E. Punta, and E. Usai, "A survey of applications of second-order sliding mode control to mechanical systems," Int. J. Control, vol. 76, no. 9/10, pp. 924-941, Jun. 2003.
[11] J. Davila, L. Fridman, and A. Levant, "Second-order sliding-mode observer for mechanical systems," IEEE Trans. Autom. Control, vol. 50, no. 11, pp. 785-1789, Nov. 2005.

[12] G. Bartolini, A. Pisano, and E. Usai, "Output-feedback control of container cranes: A comparative analysis," Asian J. Control, vol. 5, no. 4, pp. 825-837, Dec. 2003.

[13] Y. Fang, W. E. Dixon, D. M. Dawson, and E. Zergeroglu, "Nonlinear coupling control laws for an underactuated overhead crane system," IEEE/ASME Trans. Mechatron., vol. 8, no. 3, pp. 418-423, Sep. 2003.

[14] W. Singhose, K. L. Sorensen, and S. Dickerson, "A controller enabling precise positioning and sway reduction in bridge and gantry cranes," Control Eng. Pract., vol. 15, no. 7, pp. 825-837, Jul. 2007.

[15] F. Hamerlain, K. Achour, T. Floquet, and W. Perruquetti, "Higher order sliding mode control of wheeled mobile robots in the presence of sliding effects," in Proc. 44th IEEE Conf. Decision Control Eur. Control Conf., Seville, Spain, Dec. 2005, pp. 1959-1963.

[16] P. Kaveh, A. Ashrafi, and Y. Shtessel, "Integral and second order sliding mode control of harmonic oscillator," in Proc. 44th IEEE Conf. Decision Control Eur. Control Conf., Seville, Spain, Dec. 2005, pp. 3941-3946.

[17] A. Pisano and E. Usai, "Output-feedback control of an underwater vehicle prototype by higher-order sliding modes," Automatica, vol. 40, no. 9, pp. 1525-1531, Sep. 2004.

[18] S. Laghrouche, F. Plestan, and A. Glumineau, "Multivariable practical higher order sliding mode control," in Proc. 44th IEEE Conf. Decision Control Eur. Control Conf., Seville, Spain, Dec. 2005, pp. 1252-1257.

[19] D. Galzi and Y. Shtessel, "UAV formations control using high order sliding modes," in Proc. Amer. Control Conf., Minneapolis, MN, Jun. 2006, pp. 4249-4254.

[20] M. Defoort, F. Nollet, T. Floquet, and W. Perruquetti, "Higher order sliding mode control of stepper motor," in Proc. 45th IEEE Conf. Decision Control, San Diego, CA, Dec. 2006, pp. 4002-4007.

[21] M. Defoort, T. Floquet, A. Kokosy, and W. Perruquetti, "Finite-time control of a class of MIMO nonlinear systems using high order integral sliding mode control," in Proc. Int. Workshop Variable Structure Syst., Alghero, Italy, Jun. 2006, pp. 133-138.

[22] D. Galzi and Y. Shtessel, "Closed-coupled formation flight control using quasi-continuous high-order sliding mode," in Proc. Amer. Control Conf., New York, Jul. 2007, pp. 1799-1804.

[23] F. Esfandiari and H. K. Khalil, "Output feedback stabilization of fully linearizable systems," Int. J. Control, vol. 56, pp. 1007-1037, 1992.

[24] H. K. Khalil, "Robust servomechanism output feedback controllers for a class of feedback linearizable systems," Automatica, vol. 30, pp. 15871599,1994

[25] A. Dabroom and H. K. Khalil, "Numerical differentiation using high-gain observers," in Proc. 37th IEEE Conf. Decision Control, San Diego, CA, Dec. 1997 , pp. 4790-4795.

[26] A. N. Atassi and H. K. Khalil, "A separation principle for the stabilization of a class of nonlinear systems," IEEE Trans. Autom. Control, vol. 44, no. 9, pp. 1672-1687, Sep. 1999.

[27] M. Krstic, I. Kanellakopoulos, and P. V. Kokotovic, Nonlinear and Adaptive Control Design. New York: Wiley, 1995.

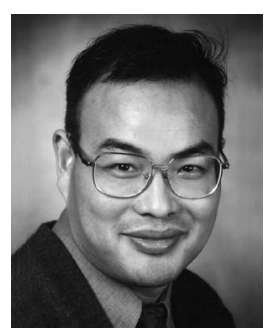

Weitian Chen (M'08) received the B.S. degree in mathematics from Ludong University, Shangdong, China, in 1989, the M.S. degree in control theory from Qufu Normal University, Shangdong, in 1991, and $\mathrm{Ph} . \mathrm{D}$. degrees from Shanghai Jiaotong University, Shanghai, China, and Simon Fraser University, Simon Fraser University, Burnaby, BC, Canada, in 1996 and 2007, respectively.

From 1996 to 1998, he was an Associate Professor and, from 1999 to 2000, a Full Professor with the Institute of Automation, Qufu Normal University. From 2001 to 2002, he was with Simon Fraser University as a Research Associate. He is currently a Research Fellow with the Department of Information Engineering, The Australian National University, Canberra, Australia. $\mathrm{He}$ has authored over 40 papers published in refereed international journals and conference proceedings. His research interests include model-based fault diagnosis in control systems, multiple model-based observation and control of uncertain linear and nonlinear systems, fuzzy systems and control, and nonlinear systems and control. 


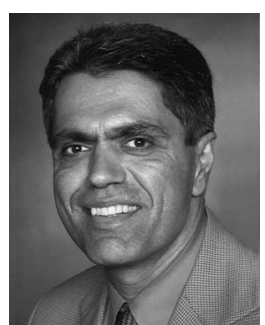

Mehrdad Saif (SM'82) received the B.S., M.S., and $\mathrm{Ph} . \mathrm{D}$. degrees in electrical engineering from Cleveland State University, Cleveland, OH, in 1982, 1984 , and 1987, respectively.

During his graduate studies, he worked on research projects sponsored by the NASA Lewis (now Glenn) Research Center, as well as the Cleveland Advanced Manufacturing Program. In 1987, he joined the School of Engineering Science, Simon Fraser University, Vancouver, BC, Canada, as an Assistant Professor, where he is currently a Full Professor and has been the Director since 2002. During 1993-1994, he was a Visiting Scholar at the General Motors North American Operation (NAO) R\&D Center, Warren, MI. At GM, he was a member of the Powertrain Control Group, Electrical and Electronics Research Department, where he worked on engine control and on-board engine diagnostic problems. He has been a Consultant to a number of industries and agencies such as GM, NASA, B.C. Hydro, Ontario Council of Graduate Studies, etc. He is an Associate Editor for the International Journal of Control and Computers. He has authored over 150 papers published in refereed journals and conference proceedings and edited a book. His research interests are in systems and control, particularly estimation and observer theory, model-based fault diagnostics in control systems, and application of these to automotive, power, and other complex engineering systems.

Dr. Saif is a Registered Professional Engineer in the Province of British Columbia, Canada. He served two terms (1995 and 1997) as the Chairman of the Vancouver Section of the IEEE Control Systems Society. He is an Associate Editor for the IEEE SYSTEMS JOURNAL. He is also on the Editorial Board of the American Control Conference, as well as the IEEE Conference on Decision and Control. 